Onkologie 1987;10(suppl 2):2

\title{
Impressum, Vol. 10, Supplement 2, 1987
}

\section{s. karger}

Verlag für Medizin und Naturwissenschaften GmbH, Postfach 1724, D-8034 Germering

Anzeigen

S. Karger Verlag für Medizin und Naturwissenschaften GmbH

Postfach 1724, D-8034 Germering, Telefon (089) 844021.

Für den Inhalt außerhalb des redaktionellen Teiles (insbesondere Anzeigen,

Industrieinformationen, Pressezitate und Kongreß-informationen) übernehmen Schriftleitung,

Beirat und Verlag kei-ne Gewähr.

Eine Markenbezeichnung kann warenzeichenrechtlich geschützt sein, auch wenn bei ihrer Verwendung in dieser Zeitschrift das Zeichen ${ }^{\circledR}$ oder ein anderer Hinweis auf etwa bestehende Schutz-rechte fehlen sollte. Für Satzfehler, insbesondere bei Dosierungs-angaben, wird keine Gewähr übernommen.

Alle Rechte, insbesondere das Recht der Vervielfältigung und Mikrokopie sowie der Übersetzung, vorbehalten. Nachdruck, auch auszugsweise, nur mit Genehmigung des Verlages. (C) Copyright 1987 by S. Karger Verlag für Medizin und Naturwissenschaften GmbH, Postfach 1724, D-8034 Germering

Satz und Druck: Walter Biering GmbH, Grafíscher Betrieb, D-8000 München 45

Redaktionelle Bearbeitung und Zusammenstellung dieser Beilage: Dr. med. E. G. Coeugniet, Bad Bentheim

Wir danken nachfolgenden Firmen für ihre Unterstützung bei der Herstellung dieser Beilage: biosyn Arzneimittel GmbH, Stuttgart

Delalande GmbH, Köln

Helixor Heilmittel GmbH, Rosenfeld

Dr. Madaus GmbH \& Co, Köln

Institut Mérieux GmbH, Norderstedt

Novipharm GmbH, Pforzheim

Schaper \& Brümmer GmbH \& Co KG, Salzgitter

Serono Pharm. Präparate GmbH, Freiburg 1987;010:2 